\title{
Estudio Comparativo entre Terapia con Melissa officinalis vs. Tratamiento Convencional con Aciclovir Tópico al $5 \%$ para Lesiones Herpéticas Tipo 1
}

\author{
Comparative Study of Therapy with Melissa Officinalis vs. Conventional \\ Topical Treatment with $5 \%$ Acyclovir in Type 1 Herpes Lesions
}

Fernanda Amin B. ${ }^{*}$ \& Patricio Oliva M. ${ }^{*}$

\begin{abstract}
AMIN, B. F. \& OLIVA, M. P. Estudio comparativo entre terapia con Melissa officinalis vs. tratamiento convencional con aciclovir tópico al 5\% para lesiones herpéticas tipo 1. Int. J. Odontostomat., 8(3):323-328, 2014.

RESUMEN: El herpes simple tipo I es una patología infecciosa e inflamatoria de tipo vírico que se caracteriza por la aparición de lesiones ulcerativas extra orales, de duración aproximada de 14 días y que provocan gran picazón e incomodidad a las personas que lo padecen. El objetivo de este trabajo fue evaluar el uso la Melissa officinalis (toronjil) como terapia alternativa sobre el tiempo de remisión y el número de las lesiones extraorales en pacientes con herpes simple del comparado con el Aciclovir tópico al 5\% como terapia convencional. Se realizó un ensayo clínico, aleatorizado y enmascarado simple, controlado. Se evaluó a 34 pacientes con lesiones herpéticas extra orales activas en la etapa primaria vesiculosa, sin patologías asociadas a alteraciones del sistema inmunológico como diabetes mellitus, alergias e hipertensión arterial. Se les administró el tratamiento tópico y se les evalúo diariamente las lesiones herpéticas. La Melissa officinalis posee un efecto antiviral sobre lesiones herpéticas tipo 1 al igual que el Aciclovir tópico al 5\%, pero la Melissa officinalis es más efectivo en la disminución de días con lesiones herpéticas (valor $p=0,05$ ). La Melissa officinalis tiene un efecto antiviral positivo sobre lesiones herpéticas tipo 1, disminuyendo el número total de días con las lesiones herpéticas en comparación con el Aciclovir tópico al $5 \%$.
\end{abstract}

PALABRAS CLAVE: herpes simple tipo 1, patología viral, lesiones ulcerativas, Melissa officinalis, aciclovir tópico al 5\%, antiviral.

\section{INTRODUCCIÓN}

El herpes simple es una enfermedad infecciosa inflamatoria de tipo vírico, que se caracteriza por la aparición de lesiones cutáneas formadas por pequeñas vesículas agrupadas en racimo y rodeadas de un halo rojo (Sapp et al., 1998). Es causada por el virus herpes hominis de tipo I (VHS-1) y puede erupcionar en cualquier parte del cuerpo, particularmente en la boca y los genitales acompañada de ardor y prurito (Manzur et al., 2002), aunque en su expresión más compleja puede derivar en fiebre, confusión, coma y signos neurales focalizados (Reyes Romero et al., 2012)

El herpes simple (HVS) es un virus de cadena simple de ADN (Arduino \& Porter, 2006) rodeada de capas de proteínas y una envoltura de lipoproteínas de una forma esférica con un diámetro de 150 a 200 $\mathrm{nm}$ (Reyes Romero et al.) es de carácter neurotrópico, el cual infecta nervios periféricos y emigra a un ganglio nervioso regional donde permanece en estado de latencia encontrándose protegido del sistema inmune y agentes terapéuticos (Arduino \& Porter).

La infección por VHS-tipo 1 ocurre normalmente en la niñez y la seropositividad aumenta con la edad, desarrollándose en un $30 \%$ a $80 \%$ de la población mundial (Smith \& Robinson, 2002). El ser humano es su huésped natural, transmitiéndose por contacto directo desde la piel erosionada o saliva (Fitzpatrick et al., 1992). 
Entre las terapias utilizadas para la remisión del Herpes Simple se encuentra el Aciclovir tópico al 5\% tópico o en tabletas (Nolkemper et al., 2006), que inhibe la replicación viral y con esto el progreso de las lesiones, utilizándose de 5 a 7 días (dependiendo de la severidad de la lesión) aplicándose de 3 a 4 veces al día, sus efectos adversos son reacciones de hipersensibilidad (alergia), erosión en la piel, dificultad para respirar, interacciona con otros medicamentos utilizados para enfermedades cardiovasculares, del hígado y riñón (Yeung-Yueet et al., 2002).

Dado lo anterior, se ha generado una línea investigativa en base a fitofármacos (Taschieri \& Del Fabbro, 2011) que evitan las complicaciones asociadas al consumo continuo de medicamentos (Howe, 1962; Regalado Veloz et al., 2012). Los fitofármacos utilizados en odontología se encuentran la manzanilla, evaluado como colutorio antibacteriano (Gaete \& Oliva, 2012), Ilantén, Aloe vera como cicatrizantes entre otros (Neumann Fumeron \& Oliva Mella, 2003).

La Melissa officinalis es una planta proveniente del sur de Europa (Kucera et al., 1965), pertenece a la familia Lamiaceae, y se compone por ácidos hydroxycinámicos y los aceites esenciales, especialmente por terpenoides como el citral, citronelal, geraniol, nerol y linalol (Esquivel \& Vargas, 2007), además posee flavonoides y taninos (Stashenko et al., 2006). El rendimiento de la extracción y calidad del aceite esencial de $M$. officinalis depende del ambiente y del periodo en el cual son cosechadas las hojas (Saeb \& Gholamrezaee, 2012).

La Melissa officinalis posee un efecto antiviral (Dimitrova et al., 1993; Allahverdiyev et al., 2004), además de poseer propiedades antibacterianas y antimicóticas (Mimica-Dukic et al., 2004), evaluando su efecto antitumoral, debido a sus componentes antioxidantes (de Sousa et al., 2004), así como su capacidad sedante (Sadraei et al., 2003).

En la presente investigación se comparó el uso de terapia con crema en base a extracto de Melissa officinalis utilizado de forma tópica comparándolo con el tratamiento convencional (Aciclovir tópico al 5\% tópico) para la evaluación del efecto antiviral en el tratamiento del virus herpes tipo 1 en relación al tiempo y número de vesículas en boca, en pacientes de la Clínica Odontológica de la Universidad del Desarrollo mediante la utilización de un escenario clínico controlado, por lo que se establece como objetivo
Determinar la eficacia de la crema de Melissa officinalis para la remisión de herpes simple comparado con Aciclovir tópico al $5 \%$ en pacientes adultos de la facultad de odontología de la Universidad del Desarrollo en Concepción, Chile.

\section{MATERIAL Y MÉTODO}

El diseño es un ensayo clínico aleatorizado con enmascaramiento simple (cegando a los pacientes), controlado con un grupo que utiliza la crema de extracto de Melissa officinalis, y otro grupo de control que ocupa Aciclovir tópico al $5 \%$ tópico para la remisión de las lesiones producidas por el herpes simple.

La muestra se calcula a partir del universo de pacientes con dicha condición en el periodo de tiempo de 30 días siendo 120 sujetos. El cálculo de muestra se realiza mediante análisis de varianzas aceptando un riesgo alfa de 0,05 y un riesgo beta de 0,2 en un contraste bilateral, precisando 17 sujetos en cada grupo para detectar una diferencia mínima de 2 entre dos grupos, asumiendo queexisten 2 grupos y una desviación estándar de 2,5. Se ha estimado una tasa de pérdidas de seguimiento del $0 \%$.

Los individuos fueron reclutados en la clínica odontológica de la Facultad durante el año 2013. Se efectuó un muestreo probabilístico por conglomerado manteniendo una relación 1:1, un grupo de intervención y un grupo placebo (Fig. 1). Los individuos tienen una edad promedio de 36 años, manteniendo una proporcionalidad entre hombres y mujeres.

Se aleatorizó por bloques por un asignador externo en dos grupos, generando un código con el cual se etiquetó los frascos correspondientes por un asignador manteniendo el ocultamiento al odontólogo interventor. La generación de número aleatorios se efectuó en el programa Excel $2010 \AA$. El listado de aleatorización fue entregado a un asignador diferente al odontólogo del tratamiento. Se mantuvo oculta la secuencia de aleatorización a los investigadores que atendían a los pacientes

La elaboración de la crema de Melissa officinalis se realizó con una base de aceite de maravilla (600 ml), al cual se le incorporó hierba de Melissa officinalis macerada $(300 \mathrm{~g})$ por 20 días obteniendo así el principio activo de la planta. Luego se unió este 


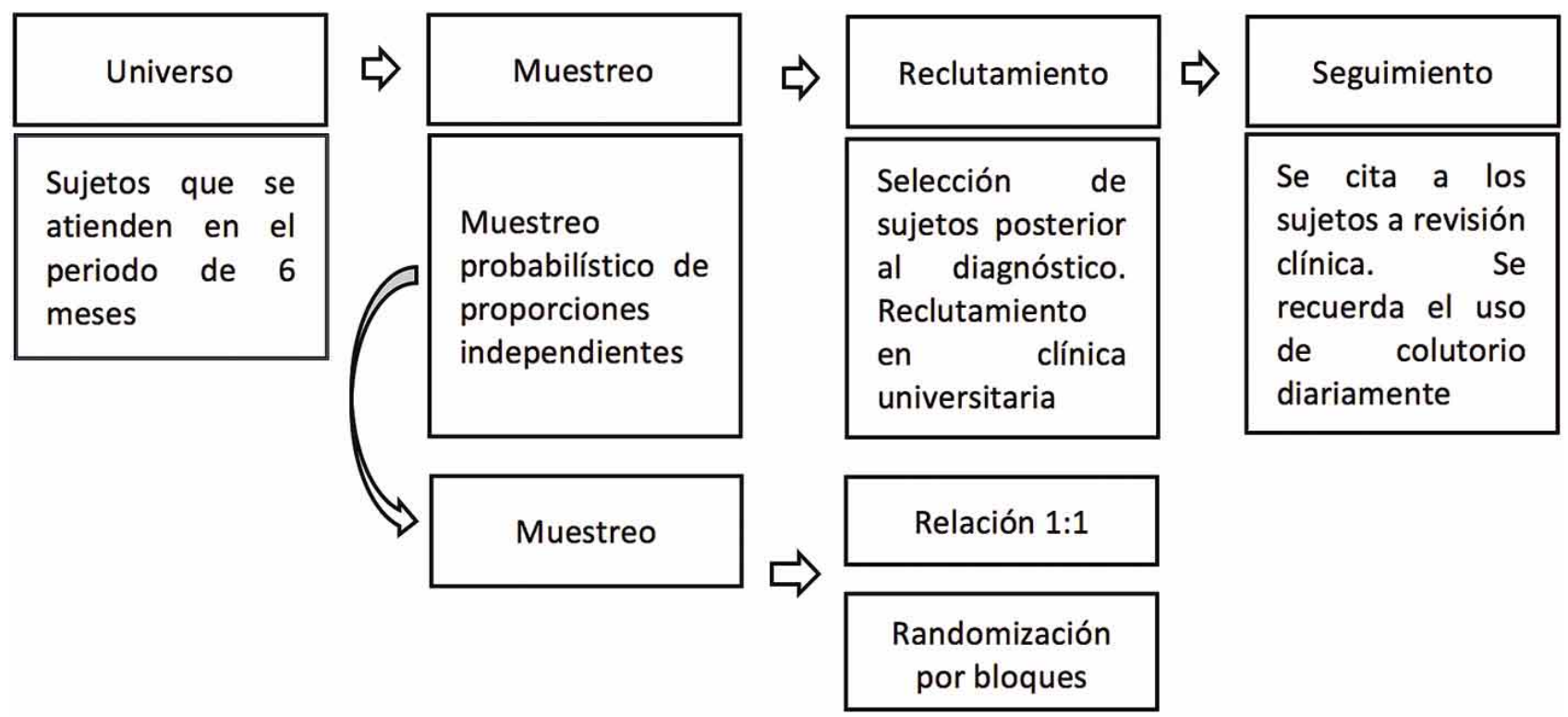

Fig. 1. Descripción de la muestra.

extracto con la vaselina solida $(400 \mathrm{~g})$ y se adicionó 5 gramos de vitamina $\mathrm{C}$. Se mezcló hasta crear un crema, la cual posteriormente fue envasada y distribuida. Se les indicó la utilización de la crema 3 veces al día, enseñándoles la porción y forma de aplicación.

Se evaluó a 34 pacientes con lesiones herpéticas extra orales activas en la etapa primaria vesiculosa, que consultados previamente no presentaron patologías asociadas a alteraciones del sistema inmunológico como diabetes mellitus, alergias e hipertensión arterial. Luego se realizó el tratamiento que consiste en la aplicación del extracto de Melissa oficinales en crema o del Aciclovir tópico al 5\%, la aplicación es cada 4 horas hasta la remisión completa de la lesión, evaluando a los pacientes diariamente, registrando en una tabla de datos el número de lesiones y los días que esta tarda en remitir.

\section{Procedimiento estadístico}

Las variables evaluadas en este estudio fueron el tiempo de remisión de lesiones extraorales del herpes simple tipo I, para el cual se usó como indicador para medir esta variable los días. La segunda variable es el número de lesiones del Herpes extraoral, el indicador para medir esta variable fueron números $(1,2$, $3 . .$.$) . El outcome se define como la remisión de la le-$ sión herpética y la evaluación implica un conteo diario por parte del odontólogo en clínica.
Se registraron los datos en el programa SPSS $17 \circledR$ en el cual se efectuaron los siguientes pasos:

a) Estadística descriptiva para el análisis de las medias y desviación estándar.

b) Evaluación de la normalidad de las diferencias mediante la prueba de Shapiro Wilk.

c) Estadística Inferencial para el análisis de las diferencias utilizando la prueba de Wilcoxon (para variables no paramétricas). Prueba de efectos intersujetos de Greenhouse-Geisser para la evaluación de diferencias en el tiempo entre ambos tratamientos.

\section{Aspectos bioéticos}

La investigación fue revisada y aprobada por el comité de bioética de la Facultad de Odontología de la Universidad de Desarrollo en Concepción en las fase de protocolo y proyecto final. Los procedimientos realizados estuvieron regidos bajo las normas éticas de la Declaración de Helsinki de 2000 ya que todos los pacientes fueron previamente informados del procedimiento con la entrega del consentimiento informado en el cual se detalló la finalidad de la investigación. Aprobado esto se realizó la ficha clínica la cual fue un instrumento de recolección de datos validado por 5 expertos. 


\section{RESULTADOS}

Se definen dos grupos de 17 sujetos cada uno en el que se evalúa la eficacia de la Melissa officinalis comparado con Aciclovir tópico al $5 \%$ tópico (como gold standar) para la remisión de Herpes Simple. La línea de base para el grupo que utiliza la crema de extracto de Melissa officinalis establece como media 4,6 lesiones herpéticas en los sujetos en estudio con una desviación estándar de 0,3 y para el grupo que utiliza Aciclovir tópico al 5\% tópico una media de 6 lesiones herpéticas con una desviación estándar de 0,6 (Tabla I).

Tabla I. Promedios inicial de lesiones herpéticas.

\begin{tabular}{lccc}
\hline Tratamiento & $\mathbf{n}$ & $\begin{array}{c}\text { Media de } \\
\text { lesiones de } \\
\text { herpes labial }\end{array}$ & DE \\
\hline Aciclovir tópico al 5\% & 34 & 6,0 & 0,6 \\
Melissa officinalis & 34 & 4,6 & 0,3 \\
\hline
\end{tabular}

El promedio inicial de lesiones herpéticas diarias en el grupo que utilizó la crema de Melissa officinalis es de 3,9 lesiones, un valor más elevado que en el grupo que utilizó Aciclovir tópico al $5 \%$ que tuvo un media de 2,9 lesiones. Al tercer día de utilización, el grupo que utiliza Melissa officinalis disminuye el número de lesiones comparado con el grupo que utiliza Aciclovir tópico al $5 \%$ tópico, baja que se mantiene hasta sexto día donde alcanzó una media de 0,1 lesiones. El grupo que utiliza la crema de Aciclovir tópico al 5\% Tópico mantiene lesiones hasta el día 9 con una media de 0,1 lesiones (Fig. 2).
Se evaluó la normalidad de la distribución de los sujetos mediante la prueba de Shapiro-Wilk dado que la frecuencia es menor a 30 casos, y arrojó una significancia de $p<0,04$, lo que resulta en una distribución no paramétrica, por lo cual el análisis estadístico se establece dentro de dichos parámetros.

Al evaluar la diferencia significativa, mediante la prueba de los rango de los signos de Wilcoxon, entre el número de lesiones del grupo que utilizó crema de Melissa officinalis comparado con el grupo que utilizó crema con Aciclovir tópico al $5 \%$, se observa que en los primeros 4 días de tratamiento no existió diferencia estadísticamente significativa entre ambos grupos, lo que implica que los tratamientos no poseen un efecto clínico similar para dicho periodo.

En los días 5 y 6 del tratamiento existen diferencias significativas entre ambos grupos $(p<0,02)$, lo que implica que un grupo fue clínicamente superior al reducir el número de herpes en los sujetos de prueba, y que de acuerdo a la gráfica anterior es el grupo que utilizó crema de Melissa officinalis (Tabla II).

Cuando se evalúa la diferencia entre cada tiempo de medición con la prueba de efectos intersujetos de Greenhouse-Geisser para todo el tratamiento se observó una diferencia significativa entre ambos grupos $(p<0,05)$, es decir, la Melissa officinalis demuestra mejores resultados clínicos en las evaluaciones efectuadas diariamente relacionada con el número de días con lesiones herpéticas comparado con el Aciclovir tópico al 5\%.

Tabla II. Lesiónes herpéticas según día de tratamiento.

\begin{tabular}{ccccccc}
\hline & Día 1 & Día 2 & Día 3 & Día 4 & Día 5 & Día 6 \\
\hline Valor p & 0,28 & 0,74 & 0,64 & 0,41 & 0,02 & 0,02
\end{tabular}

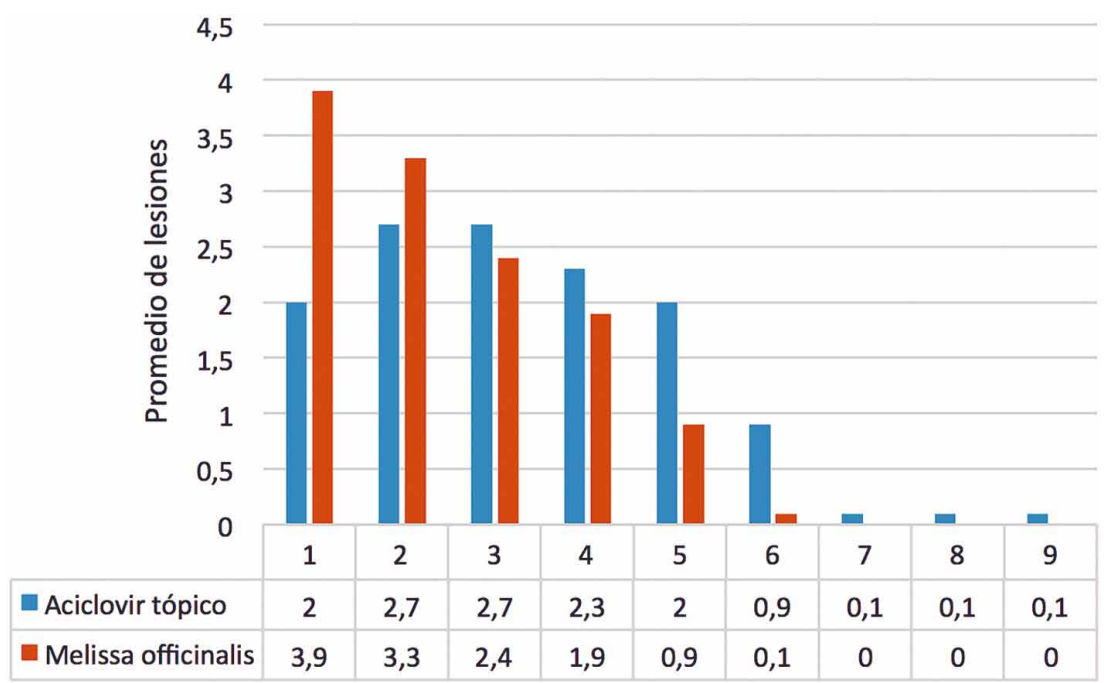

Fig. 2. Promedio de lesiones herpéticas según día de tratamiento. 


\section{DISCUSIÓN}

La investigación en productos derivados de extractos de plantas ha aumentado consistemente a lo largo del tiempo en odontología particularmente por las múltiples propiedades benéficas que contienen además de la baja o nula toxicidad, comparado con compuestos farmacológicos.

La utilización de estos productos naturales en odontología varían en el vehículo de aplicación, centrándose principalmente en la utilización de colutorios (López Jornet et al., 2003; Bascones \& Morante, 2006; Sekino \& Ramberg, 2005) utilizando en ellos extractos de aloe vera, menta, llantén (Neumann Fumeron $\&$ Oliva Mella) entre otras plantas.

El extracto de Melissa officinalis es utilizado ampliamente para diversos procedimientos clínicos, particularmente para el control de la ansiedad y sintomatologías similares, sin embargo, sus propiedades antivirales poseen un efecto de remisión sobre el VHS-1 y HSV-2 (Nolkemper et al.), siendo evaluado en investigaciones microbiológicas previas (Koytchev et al., 1999), aunque generalmente en estudios in vitro (Astani et al., 2012)

La utilización del extracto de Melissa officinalis en crema para la evaluación de la remisión del herpes simple evidenció la disminución de los días de infec- ción de forma sostenida en el periodo de evaluación, coincidiendo con investigaciones que sitúan dicho efecto a los 2 días de uso (Koytchev et al.) extiendo los resultados a la sintomatología asociada.

Investigaciones sobre la Melissa officinalis comparan el efecto antiviral en herpes simple con placebo para la evaluación del tratamiento estableciendo la eficacia de extracto de planta para la remisión del evento (Wölbling \& Leonhardt, 1994). Al comparar el mismo efecto con un gold estándar, como el Aciclovir tópico al $5 \%$ de uso tópico usado en el estudio, se mantiene la eficacia evaluando el número de días con lesiones, estableciendo un primer ciclo que homologa el efecto farmacológico del Aciclovir tópico al $5 \%$, y un segundo ciclo en el cual el Melissa officinalis disminuye el total de días (Sepúlveda Tabache et al., 2003).

Como resultado se establece que la utilización de la Melissa officinalis favorece la remisión del herpes simple disminuyendo el número de días con la patología, y demuestra un comportamiento superior al Aciclovir tópico al $5 \%$ a partir del tercer día para la remisión del mismo. Su utilización permanente de acuerdo a las instrucciones establecidas en el diseño de investigación permite el control clínico de esta condición, sumado a la ausencia de efectos adversos observados en el periodo de investigación.

AMIN, B. F. \& OLIVA, M. P. Comparative study of therapy with Melissa officinalis vs. conventional topical treatment with $5 \%$ acyclovir in type 1 Herpes lesions. Int. J. Odontostomat., 8(3):323-328, 2014.

ABSTRACT: Herpes simplex type I is an infectious viral and inflammatory disease type which is characterized by the appearance of extra- oral ulcerative lesions of approximately 14 days that cause severe itching and discomfort for those who have the lesions. The objective of this study was to evaluate the use of Melissa officinalis (lemon balm) as an alternative therapy on remission time and the number of extra oral lesions in patients with herpes simplex compared with topical $5 \%$ acyclovir and conventional therapy. A clinical, randomized and single-blind, controlled trial study was carried out. Thirty-four patients were evaluated with active extra- oral herpetic vesicular lesions in primary stage, without pathologies associated with immune system disorders such as diabetes mellitus, allergies and hypertension. They were given topical treatment and herpetic lesions were evaluated daily. Melissa officinalis has an antiviral effect on type 1 herpes lesions as $5 \%$ topical acyclovir, Melissa officinalis but is more effective in the reduction of days with herpetic lesions ( $p$ value $<0.05$ ). In conclusion, Melissa officinalis has a positive antiviral effect on type 1 herpes lesions, reducing the total number of days with herpetic lesions compared with topical $5 \%$ acyclovir.

KEY WORDS: herpes simplex type 1, viral disease, ulcerative lesions, Melissa officinalis, topical acyclovir, antiviral.

\section{REFERENCIAS BIBLIOGRÁFICAS}

Allahverdiyev, A.; Duran, N.; Ozguven, M. \& Koltas, S. Antiviral activity of the volatile oils of Melissa officinalis L. against Herpes simplex virus type-2. Phytomedicine, 11(7-8):65761, 2004.
Arduino, P. G. \& Porter, S. R. Oral and perioral herpes simplex virus type 1 (HSV-1) infection: review of its management. Oral Dis., 12(3):254-70, 2006. 
AMIN, B. F. \& OLIVA, M. P. Estudio comparativo entre terapia con Melissa officinalis vs. tratamiento convencional con aciclovir tópico al $5 \%$ para lesiones herpéticas tipo 1. Int. J. Odontostomat., 8(3):323-328, 2014.

Astani, A.; Reichling, J. \& Schnitzler, P. Melissa officinalis extract inhibits attachment of herpes simplex virus in vitro. Chemotherapy, 58(1):70-7, 2012.

Bascones, A. \& Morante, S. Antisépticos orales: Revisión de la literatura y perspectiva actual. Av. Periodoncia Implantol. Oral, 18(1):21-9, 2006.

de Sousa, A. C.; Alviano, D. S.; Blank, A. F.; Alves, P. B.; Alviano, C. S. \& Gattass, C. R. Melissa officinalis L. essential oil: antitumoral and antioxidant activities. J. Pharm. Pharmacol., 56(5):677-81, 2004.

Dimitrova, Z.; Dimov, B.; Manolova, N.; Pancheva, S.; llieva, D. \& Shishkov, S. Antiherpes effect of Melissa officinalis L. extracts. Acta Microbiol. Bulg., 29:65-72, 1993.

Esquivel, F. A. \& Vargas, P. Uso de aceites esenciales extraídos por medio de fluidos supercríticos para la elaboración de alimentos funcionales. Tecnol. Marcha, 20(4):41-50, 2007.

Fitzpatrick, T. B.; Johnson, R. A.; Polano, M. K.; Suurmond, D. \& Wolff, K. Atlas de dermatología clínica. Madrid, Ediciones Doyma, 1992.

Gaete, M. \& Oliva, P. Effectiveness of chamomile mouthwash compared with placebo and chlorhexidine in patients with gingivitis aged 19 to 25 years: controlled clinical trial. Int. J. Odontostomatol., 6(2):151-6, 2012.

Howe, G. L. Some complications of tooth extraction. Ann. R. Coll. Surg. Engl., 30:309-23, 1962.

Kucera, L. S.; Cohen, R. A. \& Herrmann, E. C. Jr. Antiviral activities of extracts of the lemon balm plant. Ann. N. Y. Acad. Sci., 130(1):474-82, 1965.

Koytchev, R.; Alken, R. G. \& Dundarov, S. Balm mint extract (Lo-701) for topical treatment of recurring herpes labialis. Phytomedicine, 6(4):225-230, 1999.

López Jornet, P.; Henarejas Hernández, J. L.; Saura Pérez, M. \& Camacho Alonso, F. Efectos de los diferentes colutorios para el tratamiento de la halitosis oral. Av. Odontoestomatol., 19(6):275-82, 2003.

Manzur, J.; Díaz, J.; Cortés, M.; Regalado, P.; Sagaró, B. \& Abreu, A. Libro Dermatología. La Habana, Ciencias Médicas, 2002.

Mimica-Dukic, N.; Bozin, B.; Sokovic, M. \& Simin, N. Antimicrobial and antioxidant activities of Melissa officinalis L. (Lamiaceae) essential oil. J. Agric. Food Chem., 52(9):2485-9, 2004.

Neumann Fumeron, C. \& Oliva Mella, P. Efecto del Llantén mayor en la cicatrización secundaria de alvéolo post exodoncia: Estudio clínico preliminar en adultos. Acta Odontol. Venez., 51(4):1-10, 2003.

Nolkemper, S.; Reichling, J.; Stintzing, F. C.; Carle, R. \& Schnitzler, P. Antiviral effect of aqueous extracts from species of the Lamiaceae family against Herpes simplex virus type 1 and type 2 in vitro. Planta Med., 72(15):1378-82, 2006.
Regalado Veloz, A. I.; Sánchez Perera, L. M. \& Mancebo Dorvigny, B. Tratamientos convencionales y medicina alternativa de la úlcera péptica. Rev. Cubana Farm., 46(1):127-37, 2012.

Reyes Romero, H.; Rojas Navarro, P.; Porta González, L.; Klein Palmero, K.; de la Parte, M. A.; Barrios Reyes, H. Actualización en infecciones por el virus Herpes Simplex. Inf. Méd., 14(8):373, 2012.

Saeb, K. \& Gholamrezaee, S. Variation of essential oil composition of Melissa officinalis $L$. leaves during different stages of plant growth. Asian Pac. J. Trop. Biomed., 2(1):547-9, 2012.

Sapp, J. P.; Eversole, L. R. \& Wysocki, G. P. Patología Oral y Maxilofacial Contemporánea. Madrid, Elsevier, 1998.

Sadraei, H.; Ghannadi, A. \& Malekshahi, K. Relaxant effect of essential oil of Melissa officinalis and citral on rat ileum contractions. Fitoter., 74(5):445-52, 2003.

Sepúlveda Tabache, E.; Brethauer Meier, U.; Jiménez Moraga, M.; Morales Figueroa, R.; Rojas Castro, J. \& Le Fort Canales, P. Detección del virus herpes simple en lesiones de la mucosa oral en pacientes con terapia oncológica. Med. Oral, 8:32933, 2003.

Sekino, S. \& Ramberg, P. The effect of a mouth rinse containing phenolic compounds on plaque formation and developing gingivitis. J. Clin. Periodontol., 32(10):1083-8, 2005.

Smith, J. S. \& Robinson, N. J. Age-specific prevalence of infection with herpes simplex virus types 2 and 1: a global review. $J$. Infect. Dis., 186(Suppl. 1):S3-28, 2002.

Stashenko, E. E.; Jaramillo, B. E. \& Martínez, J. R. Comparación de la composición química y de la actividad antioxidante in vitro de los metabolitos secundarios volátiles de plantas de la familia Verbenaceae. Rev. Acad. Colomb. Cienc., 27(105):57997, 2003.

Taschieri, S. \& Del Fabbro, M. Postextraction osteotome sinus floor elevation technique using plasma-rich growth factors. Implant Dent., 20(6):418-24, 2011.

Wölbling, R. H. \& Leonhardt, K. Local therapy of herpes simplex with dried extract from Melissa officinalis. Phytomedicine, 1(1):25-31, 1994.

Yeung-Yue, K. A.; Brentjens, M. H.; Lee, P. C. \& Tyring, S. K. The management of herpes simplex virus infections. Curr. Opin. Infect. Dis., 15(2):115-22, 2002.

Dirección para Correspondencia:

Patricio Oliva

Barros Arana 1734

Concepción

CHILE

Email: patriciooliva@udd.cl

Recibido : 28-04-2014

Aceptado: 13-11-2014 